\title{
Avaliação da deformidade estética após a tenotomia da cabeça longa do bíceps na artroscopia do ombro*
}

\author{
Assessment of esthetic deformity after tenotomy of \\ the long head of the biceps in shoulder arthroscopy \\ Alexandre AlmeidA ${ }^{1}$, Gilberto Roveda ${ }^{1}$, MÁrcio Rangel Valin ${ }^{1}$, \\ Nayvaldo Couto de Almeida ${ }^{1}$, Ana Paula AgostinI ${ }^{2}$, CAmila ScheifleR ${ }^{3}$
}

\section{RESUMO}

Objetivo: Analisar a freqüência da queixa estética resultante do procedimento da tenotomia da cabeça longa do bíceps (CLB), com o propósito de criar um critério de análise pré-operatória capaz de identificar os pacientes com tendência a apresentarem queixa estética decorrente de tal procedimento, com a intenção de reduzir sua incidência. Métodos: Foi analisado um grupo de 89 pacientes (90 ombros) submetidos à tenotomia artroscópica da CLB. Os pacientes incluídos no estudo tiveram seu índice de massa corporal (IMC) aferido e foram classificados entre os diferentes graus de obesidade. Foram aferidas a dobra cutânea abdominal e a tricipital contralateral com um adipômetro graduado em milímetros, bem como determinado o biótipo de cada indivíduo através do ângulo mensurado no apêndice xifóide. A tenotomia do CLB, quando indicada, foi realizada junto a sua inserção no labrum supe-

* Trabalho realizado no Hospital Saúde de Caxias do Sul (RS), Brasil.

1. Médico Ortopedista do Hospital Saúde de Caxias do Sul (RS), Brasil.

2. Mestre em Medicina do Hospital São Lucas da Pontíficia Universidade Católica do Rio Grande do Sul - PUCRS - Porto Alegre (RS), Brasil.

3. Psicóloga do Hospital Saúde de Caxias do Sul (RS), Brasil.

Endereço para correspondência: Rua Vitório Buzelatto, 222/601, Bairro Madureira - 95020-290 - Caxias do Sul (RS), Brasil. Tel.: 55-54-3221-4163. E-mail: ale@visao.com.br

Recebido em 23/7/07. Aprovado para publicação em 19/6/08. Copyright RBO2008 rior da glenóide. Os pacientes foram questionados quanto à percepção da deformidade residual gerada pela tenotomia do CLB. As variáveis estudadas foram: idade, sexo, lado operado, dominância, biótipo, grau de obesidade, dobra cutânea abdominal e dobra cutânea tricipital contralateral. Foram avaliados 77 pacientes com média de $15,14( \pm 10,7)$ meses de pós-operatório. Resultados: Não houve queixa da deformidade em $50(64,9 \%)$, enquanto 27 pacientes $(35,1 \%)$ manifestaram alguma queixa estética. A análise estatística não foi significativa quando avaliou de forma cruzada a idade ( $p=$ 0,788), o lado $(p=0,075)$ e o biótipo $(p=0,529)$ dos pacientes que apresentaram queixa estética de sua deformidade residual. Houve correlação significativa para a queixa da deformidade quando foram avaliados o sexo $(p=0,004)$, a dominância $(p=$ 0,026) e ao agrupar os pacientes com IMC abaixo de 30 (normal e sobrepeso) e compará-los com os pacientes com IMC acima de 30 (obesidade graus $\mathbf{1}$, 2 e mórbida) $(p=0,005)$. Demonstrou-se correlação significativa para a queixa da deformidade nos pacientes com a dobra cutânea abdominal abaixo de $23,2 \mathrm{~mm}(p=0,003)$, bem como com a dobra cutânea tricipital contralateral abaixo de $14,5 \mathrm{~mm}(p=$ 0,001). Conclusões: A freqüência da queixa estética foi de $35,1 \%$ na amostra. Pacientes masculinos; submetidos à tenotomia do CLB no lado dominante; com IMC abaixo de 30; com dobra cutânea abdominal abaixo de $23,2 \mathrm{~mm}$ e dobra cutânea tricipital contralateral abaixo de $\mathbf{1 4 , 5} \mathbf{m m}$ são considerados de 


\section{risco para apresentar queixa estética da deformi- dade residual.}
Descritores - Ombro/cirurgia; Artroscopia/efeitos adver- sos; Avaliação/métodos; Obesidade

\section{ABSTRACT}

Objective: To analyze the frequency of esthetic complaints after tenotomy of the long head of the biceps in order to create a criterion for some preoperative analysis that could identify patients that tend to complain about esthetics after the procedure, and thus reduce the incidence. Methods: A group of 89 patients (90 shoulders) submitted to arthroscopic tenotomy of the long head of the biceps was analyzed. Patients included in the study had their bodily mass index (BMI) measured and were classified into different degrees of obesity. The abdominal skin fold and the contralateral tricipital skin fold were measured with a millimeter-graded adipometer, and the biotype of each individual was determined by the angle measured in the xiphoid appendix. Tenotomy of the long head of the biceps, when indicated, was performed at its insertion in the upper labrum of the glenoid. Patients were asked about their perception of residual deformity created by the tenotomy of the long head of the biceps. Variables studied were age, gender, operated side, dominance, biotype, degree of obesity, abdominal skin fold, and contralateral tricipital skin fold. 77 patients were evaluated for a mean 15.14 ( \pm 10.7$)$ months period after the surgery. Results: 50 patients (64.9\%) did not complain about deformity, whereas 27 patients (35.1\%) had some esthetic complaint. Statistical analysis was not significant in the cross analysis for age $(P=0.788)$, the side $(P=0.075)$, and the biotype $(P=0.529)$ of the patients who did present an esthetic complaint about their residual deformity. Significant correlation of deformity complaint was present in the evaluation of gender $(p=0.004)$, dominance $(P=0.026)$, and when a group was formed of patients with BMI below 30 (normal and overweight) and this group was compared to patients with BMI above 30 (obesity grade one, two, and morbid $)(P=0.005)$. A significant correlation was shown for the deformity complaint in patients with abdominal skin fold of less than $23.2 \mathrm{~mm}(P=0.003)$, and with the contralateral tricipital skin fold of less than $14.5 \mathrm{~mm}(p=0.001)$. Conclusions: The frequency of esthetic complaint was of $35.1 \%$ in the series. Male patients; submitted to tenotomy of the long head of the biceps on the dominant side, with BMI below 30; with abdominal skin fold of less than $23.2 \mathrm{~mm}$ and contralateral tricipital skin fold of less than $14.5 \mathrm{~mm}$ are considered at risk of presenting some esthetic complaint regarding residual deformity.

Keywords - Shoulder/surgery; Artroscopy/adverse effects; Evaluation/methods; Obesity

\section{INTRODUÇÃO}

A cabeça longa do bíceps (CLB) está comprometida com frequiência nos pacientes com afecções do ombro e é reconhecida como fonte de dor ${ }^{(1-6)}$. Nos casos em que uma lesão é encontrada, ela deve ser devidamente abordada para não comprometer o resultado final do tratamento $^{(5)}$. A indicação para abordagem cirúrgica da CLB dolorosa é controversa na literatura. O desbridamento artroscópico está indicado quando há sinais de tendinite crônica ${ }^{(4,7)}$ e para as lesões com comprometimento de até $50 \%$ da espessura do tendão ${ }^{(5,8-9)}$. A tenotomia da CLB seguida ou não de tenodese está indicada para as lesões que comprometem $50 \%$ ou mais da espessura do tendão, para a instabilidade no sulco bicipital ou para o achado de lesão SLAP degenerativa em paciente idoso ${ }^{(4,7,9-10)}$.

A tática cirúrgica de liberar a CLB comprometida é reconhecida por melhorar os resultados pós-operatórios dos pacientes submetidos aos mais diversos procedimentos terapêuticos no ombro ${ }^{(11-15)}$. Resultados clínicos semelhantes podem ser alcançados apenas incluindo a CLB na sutura do manguito rotador, sem necessariamente recorrer à sua tenotomia ${ }^{(16)}$. A melhora da sintomatologia dos pacientes também é demonstrada quando a tenotomia da CLB é realizada de forma isolada, sem a realização da sutura de uma lesão do manguito rotador a ela associada ${ }^{(17-18)}$.

Independentemente da forma de como o cirurgião decide abordar a lesão da CLB, há sempre o receio de 
que a deformidade estética ou o espasmo muscular resultante da tenotomia se tornem queixas de origem iatrogênica para o paciente ${ }^{(13-15,17,19-20)}$. Esse receio está baseado no fato de que há um risco aumentado de migração distal da CLB sob efeito da tração muscular, após a tenotomia isolada da longa porção do bíceps ${ }^{(21)}$.

A deformidade estética residual é uma constante preocupação do cirurgião e costuma fazer parte das considerações pré-operatórias. Como no Serviço de Ortopedia do Hospital Saúde de Caxias do Sul/RS esse procedimento é realizado com freqüência, tal fato motivou o estudo presente.

O objetivo principal desta pesquisa é, portanto, analisar a freqüência da queixa estética resultante da tenotomia da CLB, nas síndromes dolorosas do ombro. Como objetivo secundário, tentamos criar um critério de análise pré-operatória para identificar os pacientes com potencial aumentado de apresentarem queixa estética decorrente do procedimento, com o propósito de reduzir a incidência de tal queixa.

\section{MÉTODOS}

O estudo realizado foi transversal de coorte prospectiva histórica.

Foi analisado um grupo de 89 pacientes (90 ombros) submetidos à tenotomia artroscópica da CBL no período de 29 de outubro de 2002 a 14 de fevereiro de 2007, no Hospital Saúde de Caxias do Sul.

Houve perda do acompanhamento de seis pacientes $(6,74 \%)$ da amostra inicial. Um paciente evoluiu para óbito antes da avaliação, dois pacientes não foram localizados e três abandonaram o tratamento. Foram excluídos deste estudo, no total, 13 pacientes por apresentar atrofia ou modificação estética do membro superior contralateral, comprometendo a comparação entre os membros superiores, tais como: ruptura da CLB contralateral, antecedente de fraturas e/ou cirurgias no membro superior contralateral. A média de idade dos 77 pacientes incluídos no estudo foi de 61,6 $( \pm 9,8)$ anos.

Com relação ao sexo, encontramos 24 do masculino $(31,2 \%)$ e 53 do feminino $(68,8 \%)$. O lado direito foi acometido em 67 pacientes (87\%) e o lado esquerdo, em 10 pacientes (13\%). O lado dominante foi acometido em 69 pacientes $(89,6 \%)$. Os pacientes foram submetidos à medição da altura em centímetros e do peso em quilogramas no pré-operatório imediato. Os valores encontrados foram utilizados para cálculo do $\mathrm{IMC}^{(22)}$.

Os pacientes foram classificados de acordo com o IMC entre os diferentes graus de obesidade ${ }^{(22)}$.

Com relação aos graus de obesidade, 26 pacientes foram classificados como normais $(33,8 \%), 31$ apresentavam sobrepeso $(40,3 \%), 17$ como obesidade grau um $(22,1 \%)$ e três como grau dois $(3,9 \%)$. Não houve casos de obesidade mórbida na casuística.

As espessuras da dobra cutânea abdominal e da tricipital contralateral foram aferidas com um adipômetro da marca Cescorf ${ }^{\circledR}$ graduado em milímetros. A espessura da dobra cutânea abdominal foi aferida em um ponto localizado a $2 \mathrm{~cm}$ laterais à esquerda e $2 \mathrm{~cm}$ distais à cicatriz umbilical. A espessura da dobra cutânea tricipital contralateral foi aferida com o paciente em posição ortostática, mantendo os braços estendidos e relaxados junto ao corpo, em um ponto médio localizado na face posterior do braço, entre o acrômio e o olécrano, em prega paralela ao eixo longitudinal do membro superior (MS) ${ }^{(23-24)}$.

A dobra cutânea abdominal variou entre $7 \mathrm{~mm}$ e $58,5 \mathrm{~mm}$, com a média em $26,5 \mathrm{~mm}( \pm 12,1)$. A dobra cutânea tricipital contralateral variou entre $3 \mathrm{~mm}$ e $30 \mathrm{~mm}$, com a média em $16,97 \mathrm{~mm}( \pm 7,4)$.

O biótipo foi analisado por meio do ângulo mensurado no apêndice xifóide, classificando os pacientes, de acordo com os critérios propostos por Viola e descritos por Vieira Romeiro e Porto, em: brevilíneo (ângulo $>90^{\circ}$ ), normolíneo (ângulo entre $70^{\circ}$ e $90^{\circ}$ ) e longilíneo (ângulo menor que $\left.70^{\circ}\right)^{(23-24)}$. Com relação ao biótipo, 40 pacientes eram normolíneos $(51,9 \%)$, 35 brevilíneos $(45,5 \%)$ e dois longilíneos $(2,6 \%)$.

Após realizada a anestesia, o paciente foi posicionado em decúbito lateral com o membro superior em $30^{\circ}$, flexionado em $20^{\circ}$ e sob tração de $5 \mathrm{~kg}$. A técnica de distensão articular foi o soro fisiológico em suspensão ${ }^{(25)}$. A tenotomia da CLB foi realizada com uma pinça Trimmer e junto à sua inserção no lábio superior da glenóide sempre que observado comprometimento 
de $50 \%$ ou mais da espessura tendinosa, diagnosticada instabilidade no sulco intertubercular ou encontrada SLAP degenerativa. O procedimento foi executado sempre pelo mesmo cirurgião.

Todos os pacientes foram imobilizados, ainda anestesiados, na sala cirúrgica por tipóia. Foi utilizado um coxim de abdução quando a lesão do manguito rotador suturada era grande ou extensa. No período das primeiras quatro semanas de pós-operatório, os pacientes foram orientados a evitar a flexão forçada do cotovelo, bem com sua extensão completa.

Os pacientes incluídos no estudo foram avaliados por meio de um questionário de 23 perguntas elaborado por profissional da área de psicologia. As três primeiras perguntas estão relacionadas com a percepção da deformidade residual gerada pela tenotomia da CLB. As 20 perguntas restantes têm o objetivo de identificar características psicossomáticas peculiares a determinadas personalidades (Questionário).

Neste estudo foi considerada para fins de análise estatística somente a pergunta de no 1 : "Você nota diferença entre os dois braços?" As respostas objetivas disponíveis foram: sempre, às vezes, raramente ou nunca. Os pacientes foram avaliados com média de 15,14 $( \pm 10,7)$ meses de pós-operatório, sendo o mínimo de quatro meses e o máximo de 48 meses. A mediana do período de avaliações foi de 12 meses (IIQ seis a 22 meses).

Todo paciente estudado recebeu um termo de consentimento informado aprovado pela Comissão de Ética da instituição onde o trabalho foi realizado (HSCSRS).

As variáveis estudadas foram: idade, sexo, lado operado, dominância, grau de obesidade, dobra cutânea abdominal, dobra cutânea tricipital contralateral e biótipo.

Os dados foram analisados com o pacote estatístico SPSS (Statistical Package for Social Sciences) versão 12.0 (SPSS Inc. 1989-2003). Para a análise estatística, foram utilizados: cálculo das médias, desvio-padrão, mediana, freqüência e percentual. Utilizou-se o teste de Mann-Withney para avaliação da idade. O teste exato de Fisher foi utilizado para avaliar o lado, domi-

\section{QUESTIONÁRIO}

a) Você nota diferença entre os dois braços? ( ) Sempre (1) Ảs vezes (1) Raramente ( ) Nunca

b) As pessoas percebem diferença em seus braços? ( ) Sempre (1) Às vezes (1) Raramente (1) Nunca

c) Se existe diferença, essa diferença incomoda você? ( ) Sempre (1) Às vezes (1) Raramente (1) Nunca

d) Você se sente motivado a iniciar novos projetos? ( ) Sempre (1) Às vezes (1) Raramente (1) Nunca

e) Sente mal-estar sem causa específica? ( ) Sempre (1) Às vezes (1) Raramente (1) Nunca

f) Apresenta memória e atenção alteradas?

( ) Sempre (1) Às vezes (1) Raramente ( ) Nunca

g) Fica emotivo com freqüência?

( ) Sempre (1) Às vezes (1) Raramente ( ) Nunca

h) Irrita-se facilmente quando os outros referem sua limitação?

(1) Sempre (1) Às vezes (1) Raramente ( ) Nunca

i) Perde a libido e o interesse nas pessoas e nas coisas? ( ) Sempre (1) Às vezes (1) Raramente (1) Nunca

j) Tem sonhos e pesadelos quando dorme? ( ) Sempre (1) Às vezes (1) Raramente ( ) Nunca

I) Apresenta sintomas físicos de cansaço? ( ) Sempre (1) Às vezes (1) Raramente (1) Nunca

m) Tem insônia freqüentemente e o pensamento fica confuso?

( ) Sempre (1) Às vezes (1) Raramente ( ) Nunca

n) Sente sensação de incompetência em algumas áreas? ( ) Sempre (1) Às vezes (1) Raramente (1) Nunca

o) Percebe-se com raiva desconhecendo o motivo?

( ) Sempre (1) Às vezes (1) Raramente (1) Nunca

p) Mostra-se apático, depressivo e intolerante com a vida? ( ) Sempre ( ) Às vezes (1) Raramente ( ) Nunca

q) Perde o senso de humor com facilidade e não aceita brincadeiras a seu respeito?

( ) Sempre (1) Às vezes (1) Raramente ( ) Nunca

r) Sente que os outros o olham estranho?

( ) Sempre (1) Às vezes (1) Raramente ( ) Nunca

s) Sente medo de dar andamento às coisas no geral?

( ) Sempre (1) Às vezes (1) Raramente ( ) Nunca

t) Ouve vozes com freqüência?

( ) Sempre (1) Às vezes (1) Raramente (1) Nunca

u) Consegue expressar opiniões sem preocupar-se com o que os outros pensam?

( ) Sempre (1) Às vezes (1) Raramente (1) Nunca

v) Olha-se no espelho e não gosta do que vê?

( ) Sempre (1) Às vezes (1) Raramente ( ) Nunca

$\mathrm{x}$ ) Prefere o isolamento a sair com outras pessoas ou estar em grupos?

() Sempre (1) Às vezes (1) Raramente (1) Nunca

z) Acredito que os outros não o compreendem?

( ) Sempre (1) Às vezes (1) Raramente ( ) Nunca 
nância, biótipo, IMC e medida das dobras cutâneas abdominal e tricipital contralateral. A curva ROC foi utilizada para avaliar a capacidade diagnóstica das medidas das dobras cutâneas. Foram consideradas significantes as diferenças com $\mathrm{p} \leq 0,05$ para um intervalo de confiança de $95 \%$.

\section{RESULTADOS}

O estudo avaliou um total de 77 pacientes, todos submetidos aos critérios de inclusão. Com relação à pergunta referente à deformidade, 50 pacientes $(64,9 \%)$ responderam que nunca perceberam diferença entre os dois braços. As respostas "sempre", "às vezes" ou "raramente" foram analisadas em conjunto; 27 pacientes $(35,1 \%)$ manifestaram algum grau de insatisfação de ordem estética. Quando analisamos os que apresentaram queixa estética, identificamos 20 pacientes (26\%) com queixa freqüente, respondendo "sempre" ao questionamento, e sete pacientes $(9,1 \%)$ com queixa esporádica, respondendo "às vezes" ou "raramente". Com relação à idade, não houve diferença estatística para a percepção da deformidade $(\mathrm{p}=0,788)$. Os 27 indivíduos que perceberam a diferença entre os dois membros possuíam média de idade de 61,9 $( \pm 10,9)$ anos. Os 50 indivíduos restantes, nos quais não se observou diferença significativa entre os membros, tinham média de idade de 61,5 $( \pm 9,4)$.

Houve diferença estatisticamente significativa quando foi avaliado o sexo dos pacientes da amostra em relação à presença da queixa estética $(\mathrm{p}=0,004 ; \mathrm{OR}=$ 4,3; IC95\% 1,5-12,0). Entre os homens, 58,3\% perceberam a deformidade, enquanto somente $24,5 \%$ das mulheres a registraram.

A análise estatística também não foi significativa quando avaliou de forma cruzada o lado $(\mathrm{p}=0,075)$ e o biótipo $(\mathrm{p}=0,529)$ dos pacientes que apresentaram queixa estética de sua deformidade residual. Houve diferença estatisticamente significativa quando foi avaliada a dominância em relação à presença da queixa estética ( $\mathrm{p}=0,026)$; os pacientes com o MS dominante operado apresentaram maior incidência de queixa estética.

A análise estatística demonstrou correlação significativa para a queixa da deformidade ao agrupar os pa- cientes com IMC abaixo de 30 (normal e sobrepeso) e compará-los com os pacientes com IMC acima de 30 (obesidade grau 1 e 2) $(\mathrm{OR}=7,03)(\mathrm{IC} 95 \%: 1,49-33,19)$ $(\mathrm{p}=0,005)$. Houve percepção da deformidade em 25 dos 57 pacientes $(43,9 \%)$ com IMC abaixo de 30, enquanto apenas dois (10\%) com IMC maior que 30 perceberam a deformidade.

Demonstrou-se diferença estatisticamente significativa entre os pacientes que se queixaram e os que não se queixaram da deformidade ao serem avaliadas as medidas das dobras cutâneas. Os pacientes que se queixaram da deformidade tinham média de dobra cutânea abdominal de $20,7 \mathrm{~mm}$ (mediana de $19 \mathrm{~mm}$ ) e os pacientes que não se queixaram tinham média de dobra cutânea abdominal de 29,6mm (mediana de 29mm) $(\mathrm{p}=0,002)$. A capacidade diagnóstica da medida da dobra cutânea abdominal foi avaliada por meio da curva ROC, com área de 0,714mm (IC95\% 0,594-0,833mm), sugerindo ser este um teste de média capacidade diagnóstica. $\mathrm{O}$ ponto de corte onde se encontrou a maior capacidade diagnóstica foi de $23,2 \mathrm{~mm}$, com sensibilidade de $72 \%$ e especificidade de $63 \%$. Houve percepção da deformidade em 17 dos 31 pacientes $(54,8 \%)$ com dobra cutânea abdominal abaixo de $23,2 \mathrm{~mm}$, enquanto apenas 10 dos 46 pacientes $(21,7 \%)$ com dobra maior que $23,2 \mathrm{~mm}$ perceberam a deformidade ( $\mathrm{p}$ $=0,003 ;$ OR $=4,4$; IC 95\% 1,6-11,8mm).

Os pacientes que se queixaram da deformidade tinham média de dobra cutânea tricipital de $12,8 \mathrm{~mm}$ (mediana de $11 \mathrm{~mm}$ ) e os que não se queixaram tinham média de dobra cutânea tricipital de 19,2mm (mediana de $19 \mathrm{~mm})(\mathrm{p}<0,001)$. A capacidade diagnóstica da medida da dobra cutânea tricipital contralateral foi avaliada por meio da curva ROC, com área de $0,753 \mathrm{~mm}$ (IC95\% 0,635-0,870mm), sugerindo ser este um teste de média capacidade diagnóstica. O ponto de corte, onde foi encontrada maior capacidade diagnóstica, foi de $14,5 \mathrm{~mm}$, com sensibilidade de $74 \%$ e especificidade de $67 \%$. Houve percepção da deformidade em 18 dos 31 pacientes $(58,1 \%)$ com dobra cutânea tricipital abaixo de $14,5 \mathrm{~mm}$, enquanto apenas nove dos 46 pacientes $(19,6 \%)$ com dobra maior que $14,5 \mathrm{~mm}$ perceberam a deformidade ( $\mathrm{p}=0,001 ; \mathrm{OR}=5,7$; IC95\% 2,1$15,8 \mathrm{~mm})$. 


\section{DISCUSSÃO}

Dentre as vantagens em realizar a tenotomia artroscópica da CLB ao invés de tenotomia seguida da tenodese, citam-se: a menor morbidade do procedimento, o menor número de complicações, a rapidez de realização, o fato de não interferir na reabilitação e o menor custo ${ }^{(26-27)}$. Entre as desvantagens estão citadas: a deficiência de controle de tensão na CLB com a conseqüente atrofia muscular, a perda da força de flexão e supinação do cotovelo, o surgimento de estalo no sulco intertubercular, referido por alguns pacientes como estalo doloroso (cramping pain) e a principal delas, a deformidade estética residual no braço após a migração distal da CLB tenotomizada ${ }^{(3,13,26-29)}$.

Wolf et al demonstraram, estudando cadáveres, que a migração distal da CLB ocorre com freqüência e com baixa carga de tração muscular(21). Osbahr et al ressaltaram a necessidade de orientar o paciente para evitar a flexão forçada do cotovelo e sua extensão completa no pós-operatório ${ }^{(20)}$. A observação da deformidade estética de pacientes que tiveram ruptura espontânea da CLB durante atividades diárias, comparada com a deformidade residual após a tenotomia artroscópica, sugere que o repouso orientado favorece a "autotenodese" da CLB ${ }^{(20)}$. Tivemos o cuidado de passar as mesmas orientações de repouso ao paciente.

Walch et al verificaram pouca ou nenhuma deformidade nos pacientes estudados após a liberação da CLB $^{(6)}$. Em estudo publicado em 2005, eles acompanharam o resultado de 307 tenotomias da CLB e verificaram a presença de deformidade estética significativa em 50,2\%. Não observaram deformidade em 31,9\% dos pacientes, enquanto os outros $17,9 \%$ foram classificados como duvidosos, citando entre eles pacientes obesos ou idosos com tônus muscular fraco ${ }^{(12)}$.

Boileau et al estudaram 68 pacientes (72 ombros) com lesão extensa do manguito rotador. A tenotomia artroscópica da CLB foi realizada em 39 pacientes (54\%), enquanto a tenodese foi feita em 33 (46\%). Verificaram que a prevalência da retração muscular foi significativamente maior (62\%) no grupo submetido à tenotomia da CLB sem tenodese. Nesse grupo, a defor- midade sem representar importância clínica ou estética foi percebida pelo paciente em $66,6 \%$ dos $\operatorname{casos}^{(30)}$.

Maynou et al avaliaram o resultado clínico e radiológico de 40 casos de tenotomia isolada da CLB no tratamento de lesões irreparáveis do manguito rotador e compararam com um grupo controle. Verificaram perda de $40 \%$ da força de flexão do cotovelo e notaram queixa em relação à assimetria do braço em apenas $5 \%$ da $\operatorname{amostra}^{(19)}$.

Incluímos neste estudo pacientes com todos os tipos e tamanhos de lesão do manguito rotador e não apenas lesões irreparáveis. Tivemos como objetivo principal a análise da queixa estética. Embora apontada na literatura, não consideramos a percepção da deformidade pelo profissional e sim a queixa do paciente registrada mediante respostas obtidas por meio de questionário padrão. Verificamos que havia queixa estética em $35,1 \%$ de nossa amostra.

Com relação à idade do paciente, Walch et al recomendaram não fazer a tenotomia da CLB isolada em pacientes com menos de 55 anos de idade ${ }^{(12)}$. Armstrong considerou válida a tenotomia isolada da CLB em pacientes acima dos 50 anos de idade ${ }^{(26)}$. Ball et al sugeriram evitar a tenotomia isolada da CLB em pacientes jovens ${ }^{(5)}$. Checchia et al recomendaram a tenotomia do CLB em pacientes idosos ${ }^{(18)}$. Em nossa pesquisa, não foi considerada a idade do paciente e sim a lesão da CLB para realizar a tenotomia, muito embora o paciente mais jovem do estudo tivesse 52 anos de idade. Não foi verificada diferença estatística na percepção da deformidade nas diferentes idades $(\mathrm{p}=$ 0,788).

Osbahr et al estudaram de forma comparativa a deformidade estética residual após tenotomia da CLB versus tenodese. Verificaram não haver diferença estatisticamente significativa entre os métodos e na comparação entre os sexos. Colocaram a tenotomia da CLB como alternativa razoável para abordar as afecções bicipitais $^{(20)}$.

Kelly et al verificaram a presença do "sinal de Popeye" ao repouso e durante a flexão ativa do cotovelo em 70\% dos pacientes submetidos à tenotomia da CLB. Notaram significativa predominância $(\mathrm{p}<, 05)$ do sinal nos homens $(82,7 \%)$ quando comparados com as 
mulheres $(36,5 \%)^{(13)}$. Em nosso estudo verificamos que se registrou, à semelhança do de Kelly et al, a predominância da queixa estética entre os homens ( $\mathrm{p}=$ 0,004).

Não foram encontrados na literatura trabalhos que avaliassem a relação entre a percepção da deformidade estética após tenotomia da CLB em relação com o lado operado ou com a dominância. Em nosso estudo, não foi possível demonstrar maior queixa da deformidade estética para a cirurgia realizada no lado direito ou no lado esquerdo ( $p=0,075)$, porém, com relação à dominância, observou-se que os pacientes com o lado dominante operado apresentaram maior frequiência de queixa da deformidade $(\mathrm{p}=0,026)$.

A deformidade residual após a tenotomia da CLB parece ser mais aparente em indivíduos magros e esguios. Não encontramos na literatura uma definição de quando a tenotomia pode ser realizada com relativa segurança em relação à queixa estética. Numa tentativa de eleger critérios pré-operatórios, avaliamos algumas características fenotípicas. Foram considerados: o grau de obesidade através do IMC, a dobra cutânea abdominal, a dobra cutânea tricipital e o biótipo.

Com relação ao grau de obesidade, não encontramos na literatura estudos relacionando IMC e tenotomia da CLB. Walch et al citaram a dificuldade em avaliar a deformidade estética nos indivíduos obesos ${ }^{(12)}$. Quando comparamos indivíduos magros e obesos, verificamos de forma significativa que os obesos percebem menos a deformidade $(p=0,005)$.

Nosso estudo mostrou que as medidas menores das dobras cutâneas tricipitais $(\mathrm{p}<0,001)$ e abdominais ( $\mathrm{p}$ $=0,002)$ presentes em indivíduos magros são um fator de risco para a queixa estética resultante da tenotomia da CLB.

Não foram encontrados na literatura trabalhos que avaliassem a relação entre a percepção da deformidade estética após a tenotomia da CLB com o biótipo. Em nosso estudo, não foi possível demonstrar maior queixa da deformidade estética entre os diferentes biótipos $(\mathrm{p}=0,529)$.

A utilização de um adipômetro graduado em décimo de milímetro ou um adipômetro digital que tenha capacidade de manter pressão constante durante a medida da dobra cutânea seria o ideal para o estudo. A avaliação da obesidade é mais precisa por meio da análise da gordura visceral ${ }^{(22)}$.

\section{CONCLUSÕES}

A freqüência da queixa estética esteve presente em $35,1 \%$ dos pacientes da amostra estudada que tiveram a CLB tenotomizada por via artroscópica.

Foi possível criar um critério de análise pré-operatória verificando que pacientes masculinos, submetidos à tenotomia da CLB no lado dominante, com IMC abaixo de 30, com dobra cutânea abdominal abaixo de 23,2mm e dobra cutânea tricipital contralateral abaixo de $14,5 \mathrm{~mm}$ estão mais propensos a apresentar queixa estética relacionada à deformidade residual.

\section{REFERÊNCIAS}

1. Refior HJ, Sowa D. Long tendon of the biceps brachii: sites of predilection for degenerative lesions. J Shoulder Elbow Surg. 1995;4(6):436-40

2. Boileau P, Ahrens PM, Hatzidakis AM. Entrapment of the long head of the biceps tendon: the hourglass biceps - a cause of pain and locking of the shoulder. J Shoulder Elbow Surg. 2004; 13(3):249-57.

3. Ahmad CS, ElAttrache NS. Arthroscopic biceps tenodesis. Orthop Clin North Am. 2003;34(4):499-506. Review.

4. Boileau P, Krishnan SG, Coste JS, Walch G. Arthroscopic biceps tenodesis: a new technique using bioabsorbable interference screw fixation. Arthroscopy. 2002;18(9):1002-12.

5. Ball C, Galatz LM, Yamaguchi K. Tenodesis or tenotomy of the biceps tendon: why and when to do it. Tech Shoulder Elbow Surg. 2001;2(3):140-52.

6. Walch G, Maconia G, Pozzi I, Riand N, Levigne C. Arthroscopic tenotomy of the long head of the biceps in rotator cuff ruptures. In: Gazielly DF, Gleyze P, Thomas T. The cuff. Paris: Elsevier; 1997. p. 350-5.

7. Edwards TB, Walch G. Biceps tenodesis: indications and techniques. Oper Tech Sports Med. 2002;10:99-104.

8. Richards D, Burkhart S, Lo I. Arthroscopic biceps tenodesis with interference screw fixation (The lateral decubitus position). Oper Tech Sports Med. 2003;11:15-23.

9. Richards DP, Burkhart SS. A biomechanical analysis of two biceps tenodesis fixation techniques. Arthroscopy. 2005;21(7): 861-6.

10. Sethi N, Wright R, Yamaguchi K. Disorders of the long head of the biceps tendon. J Shoulder Elbow Surg. 1999;8(7):644-54. Review. 
11. Edwards TB, Walch G, Sirveaux F, Molé D, Nové-Josserand L, Boulahia A, et al. Repair of tears of the subscapularis. Surgical technique. J Bone Joint Surg Am. 2006;88 Suppl 1 Pt 1:1-10. Review.

12. Walch G, Edwards TB, Boulahia A, Nové-Josserand L, Neyton L, Szabo I. Arthroscopic tenotomy of the long head of the biceps in the treatment of rotator cuff tears: clinical and radiographic results of 307 cases. J Shoulder Elbow Surg. 2005;14(3):238-46.

13. Kelly AM, Drakos MC, Fealy S, Taylor SA, O'Brien SJ. Arthroscopic release of the long head of the biceps tendon: functional outcome and clinical results. Am J Sports Med. 2005;33(2):208-13.

14. Gill TJ, McIrvin E, Mair SD, Hawkins RJ. Results of biceps tenotomy for treatment of pathology of the long head of the biceps brachii. J Shoulder Elbow Surg. 2001;10(3):247-9.

15. Huffman GR, Wolf EM. Arthroscopic biceps tenotomy (Results in patients with symptomatic degeneration of the long head of the biceps brachii tendon). Arthroscopy. 2003;19 (Suppl 2):5.

16. Franceschi F, Longo UG, Ruzzini L, Papalia R, Rizzelo G, Denaro V. To detach the long head of the biceps tendon after tenodesis or not: outcome analysis at the 4-year follow-up of two different techniques. Int Orthop. 2007;31(4):537-45.

17. Klinger HM, Spahn G, Baums MH, Steckel H. Arthroscopic debridement of irreparable massive rotator cuff tears - a comparison of debridement alone and combined procedure with biceps tenotomy. Acta Chir Belg. 2005;105(3):297-301.

18. Checchia SL, Doneux SP, Miyazaki AN, Fregoneze M, Silva LA, Oliveira FM, et al. Tenotomia artroscópica do bíceps nas lesões irreparáveis do Manguito rotador. Rev Bras Ortop. 2003; 38(9):513-21.

19. Maynou C, Mehdi N, Cassagnaud X, Audebert S, Mestdagh H. Clinical results of arthroscopic tenotomy of the long head of the biceps brachii in full thickness tears of the rotator cuff without repair: 40 cases. Rev Chir Orthop Reparatrice Appar Mot. 2005;91(4):300-6. French.

20. Osbahr DC, Diamond AB, Speer KP. The cosmetic appearance of the biceps muscle after long-head tenotomy versus tenodesis. Arthroscopy. 2002;18(5):483-7.

21. Wolf RS, Zheng N, Weichel D. Long head biceps tenotomy versus tenodesis: a cadaveric biomechanical analysis. Arthroscopy. 2005;21(2):182-5.

22. Mancini MC. Noções fundamentais - diagnóstico e classificação da obesidade. In: Garrido Júnior AB, editor, Ferraz EM, Barroso FL, Marchesini JB, Szego T, co-editores. Cirurgia da obesidade. São Paulo: Editora Atheneu; 2003. p. 1-7.

23. Romeiro JV. Semiologia médica. Rio de Janeiro: Guanabara Koogan; 1978.

24. Porto CC. Exame clínico - bases para a prática médica. 5a ed. Rio de Janeiro: Guanabara Koogan; 2004.

25. Almeida A, Agostini AP, Valin MR, Martins JA, Ferreira R. Artroscopia do ombro com infusão de soro fisiológico em suspensão. Estamos trabalhando de forma segura? Rev Bras Ortop. 2006;41(7):253-8.

26. Armstrong AD. Biceps tenodesis versus tenotomy. Curr Opin Orthop. 2004;15(4):239-41.

27. Gill TJ, McIrvin E, Mair SD, Hawkins RJ. Tenodesis versus release in the treatment of pathology of the long head of the biceps brachii. Presented at the Annual Meeting of the American Academy of Orthopaedic Surgeons, Orlando, FL March 2000.

28. Verma NN, Drakos M, O'Brien SJ. Arthroscopic transfer of the long head biceps to the conjoint tendon. Arthroscopy. 2005; 21(6):764.

29. Romeo AA, Mazzocca AD, Tauro JC. Arthroscopic biceps tenodesis. Arthroscopy. 2004;20(2):206-13.

30. Boileau P, Baqué F, Valerio L, Ahrens P, Chuinard C, Trojani C. Isolated arthroscopic biceps tenotomy or tenodesis improves symptoms in patients with massive irreparable rotator cuff tears. J Bone Joint Surg Am. 2007;89(4):747-57. 